\section{Desperdício de exames complementares na avaliação pré-operatória em cirurgias de catarata}

\author{
Waste of medical tests in preoperative \\ evaluation for cataract surgery
}

\author{
1 Departamento de Oftalmo- \\ Otorrinolaringologia, \\ Universidade Estadual de \\ Campinas, Campinas, Brasil. \\ Correspondência \\ Maurício Abujamra \\ Nascimento \\ Departamento de Oftalmo- \\ Otorrinolaringologia, \\ Universidade Estadual \\ de Campinas. Cidade \\ Universitária Zeferino Vaz, \\ C. P. 6.110, Campinas, $S P$ \\ 13083-970, Brasil. \\ mauabujamra@uol.com.br
}

\begin{abstract}
The objective of this clinical trial was to determine the frequency of medical tests considered unnecessary in routine preoperative evaluation for cataract surgery. Unnecessary costs with these tests were also evaluated. For patients assigned to the selective testing group, it was requested that no preoperative testing be performed unless the patient presented a new or worsening medical problem warranting medical evaluation with testing. For patients assigned to the routine testing group, three tests were requested: a 12-lead electrocardiogram, complete blood count, and serum glucose level. The costs of tests considered unnecessary were calculated. The sample of 1,025 patients consisted of 512 assigned to the routine testing group and 513 to the selective testing group. Cumulative rate of medical events was similar in the two groups ( $p=0.923)$. The selective group underwent $60.7 \%$ fewer tests. The results suggest that selective preoperative testing in cataract surgery does not harm patients in terms of peri-operative clinical complications and also represents a significant cost reduction compared to routine testing.
\end{abstract}

Cataracts; Elderly; Medical Examination; Preoperative Care
Carlos Eduardo Leite Arieta ${ }^{1}$ Maurício Abujamra Nascimento 1 Rodrigo Pessoa Cavalcanti Lira 1 Newton Kara-José 1

\section{Introdução}

Mundialmente, catarata é a causa mais prevalente de perda visual, sendo responsável por metade de todos os indivíduos cegos legalmente 1,2 . A cegueira por ela ocasionada atinge em torno de 25 milhões de pessoas 3,4, e um crescimento deste número é esperado nas próximas décadas em virtude do aumento da sobrevida da população $1,5,6$.

No momento, o único tratamento para a catarata é a cirurgia. Felizmente, a facectomia é uma das operações mais realizadas na medicina e, provavelmente, a mais efetiva 7,8. Geralmente, é um procedimento feito com anestesia local 9, sendo considerado seguro e bem tolerado pelo paciente 4,10,11,12,13. Entretanto, esta doença persiste como um importante problema de saúde pública por causa do grande número de pessoas que necessitam da cirurgia e têm dificuldade de acesso à mesma 14,15,16,17. Nos Estados Unidos, a facectomia envolve um gasto anual de 3,4 bilhões de dólares (US\$) 8,18. No Brasil, o gasto em 2001 foi de aproximadamente 110 milhões de reais, incluindo apenas as cirurgias financiadas pelo governo 19 .

Para atender às necessidades de facectomias é necessário aumentar o número de cirurgias, sem perda da qualidade mínima necessária, e reduzir os custos 14,20.

Exames subsidiários de rotina são definidos como testes solicitados a todos os pacientes a 
despeito da história clínica ou exame físico 21 . Porém, alguns estudos prévios têm sugerido que o rendimento de exames não seletivos em determinar anormalidades é baixo 9,22,23,24,25,26. Exames laboratoriais pré-operatórios podem até ser desnecessários se o paciente não tiver fatores de risco 27,28. Um fato importante é que hipertensão arterial (HAS), broncoespasmo e arritmias respondem por mais de $90 \%$ dos eventos médicos intraoperatórios na cirurgia de catarata, e testes rotineiros parecem não prevê$\operatorname{los} 9,19$.

Kaplan et al. 26, Macario et al. 29 e Mancuso 30 avaliaram protocolos de exames pré-operatórios em cirurgia geral e constataram que $40 \%$ a $70 \%$ destes exames eram desnecessários, baseando-se na história clínica e no exame físico. Como, por exemplo, mais de 250 mil facectomias são realizadas anualmente no Brasil 31,32, e 10 milhões no mundo 14, o impacto econômico potencial de racionalizar testes em países em desenvolvimento e em serviços públicos que tenham recursos financeiros limitados é significativo. Além do mais, se este conceito parece verdadeiro agora, tornar-se-á mais ainda no futuro, com o envelhecimento da população de países em desenvolvimento 1,5,6.

Dentro da rotina de um hospital universitário de referência, no Brasil, o objetivo deste experimento clínico aleatorizado foi determinar a freqüência dos exames complementares, não oculares, considerados dispensáveis numa rotina de avaliação pré-operatória de facectomias. Também foram avaliados os gastos desnecessários em relação aos custos desses exames.

\section{Metodologia}

\section{Pacientes e procedimentos médicos}

O estudo foi desenhado como um experimento clínico aleatorizado, tendo sido realizado no Hospital das Clínicas da Universidade Estadual de Campinas - UNICAMP, Campinas, São Paulo, Brasil. Essa instituição presta assistência à saúde de nível terciário, com uma demanda predominantemente composta por pacientes do sistema público de saúde da região de influência da cidade de Campinas, realizando anualmente de 2.500 a 3 mil facectomias eletivas.

Os pacientes selecionados para cirurgia de catarata foram recrutados entre 10 de fevereiro de 2000 e 10 de janeiro de 2001 . Foram excluídos do estudo pacientes com menos de 40 anos de idade, pacientes submetidos previamente a cirurgia ocular, pacientes com outras doenças oculares, como glaucoma, que necessitassem procedimentos cirúrgicos adicionais simultâneos, pacientes com indicação de anestesia geral, ou pacientes que sofreram infarto agudo do miocárdio até três meses antes da cirurgia. Todos os pacientes preencheram ficha de consentimento livre e esclarecido. O comitê de ética aprovou o protocolo geral do estudo.

A técnica cirúrgica empregada foi de extração extracapsular programada 33, e a avaliação médica pré-operatória foi realizada uma semana antes da cirurgia por um clínico geral. Foram definidos dois grupos: testes seletivos e testes rotineiros. Para os pacientes sorteados para o grupo de testes seletivos, pediu-se que nenhum teste pré-operatório fosse executado, a menos que o paciente apresentasse um novo problema médico, ou piora de uma doença préexistente, a qual requeresse os testes independentemente da realização da cirurgia. Para pacientes sorteados para o grupo de testes rotineiros, foi solicitado eletrocardiograma, dosagem de hemoglobina e glicemia de jejum. Adotou-se a classificação de condição clínica da American Society of Anesthesiologists 34 .

\section{Coleta de dados}

Os dados pré-operatórios foram coletados através de uma ficha específica contendo informações da história e exame físico, completado pelo clínico no momento da avaliação pré-operatória. Complicações clínicas intra-operatórias, ocorridas no período intra-operatório, foram registradas numa ficha de protocolo, pelo oftalmologista responsável pela cirurgia ou por um membro da equipe de enfermagem.

As definições de complicações perioperatórias registradas neste estudo estão descritas em Lira et al. 35, e são baseadas nos conceitos adotados no Current Medical Diagnosis and Treatment 36 .

As indicações dos exames complementares foram revisadas por dois investigadores (um clínico e um cardiologista) para determinar se elas se enquadravam nas diretrizes da Tabela 1 , as quais foram baseadas no protocolo proposto por Fischer 37 .

\section{Análise de gastos}

O gasto necessário para realização dos exames nos dois grupos foi baseado na tabela de valor pago pelo SUS. Para efeito de comparação, também foram calculados os gastos com os exames do total de cirurgias de catarata pagas pelo SUS. Os valores dos exames de eletrocardiograma, hemoglobina e glicemia foram agrupados num único item. Para esses exames, o valor do 
SUS foi de R $\$ 11,00$; para a cirurgia de catarata, o valor foi de $\mathrm{R} \$ 443,00$. No ano de 2001, o SUS pagou 264.406 facectomias realizadas no País.

\section{Tamanho da amostra e análise estatística}

Foi planejada uma amostra de mil pacientes (quinhentos por grupo). Assumindo-se uma taxa de eventos médicos adversos de $9 \%$ no grupo de testes rotineiros, essa amostra permitiria $90 \%$ de poder para detectar uma diferença tão pequena quanto $7 \%$ no grupo de testes seletivos. Os resultados desta análise foram considerados significantes quando o valor de $P$ foi menor que $5 \% 39,40$.

Foram feitos testes para detectar diferenças entre variáveis, usando-se o teste do qui-quadrado ou o teste exato de Fisher para variáveis categóricas, e análise de variância (one-way ANOVA), para variáveis quantitativas.

Como parâmetro de tendência central e dispersão para os dados, foi determinada a média e o desvio padrão. Executou-se a análise estatística com o auxílio do programa Epi Info (Centers for Disease Control and Prevention, Atlanta, Georgia, Estados Unidos).

\section{Resultados}

Foram incluídos 1.025 pacientes com indicação para cirurgia de catarata, com idade variando entre 40 e 97 anos, com média de 66,5 anos e desvio padrão de 11,6 anos. Houve predominância do sexo masculino, com 547 homens $(53,4 \%)$.

De acordo com os critérios de indicação de exames complementares definidos para este estudo, dos 1.025 pacientes, só haveria indicação de dosagem de hemoglobina em 37 (3,6\%) pacientes, de dosagem de glicemia de jejum em 218 (21,3\%) e realização de eletrocardiograma em 954 (93,1\%).

Vinte pacientes (2\%) faltaram à cirurgia e não a remarcaram. Dentre 1.005 pacientes que tiveram a cirurgia realizada, houve complicações clínicas intra-operatórias em 97 (9,6\%).

Os dois grupos apresentaram-se semelhantes em relação à idade, ao sexo, à classificação de condição clínica ASA e à freqüência de indicação de exames complementares (Tabelas 2 e 3).

A amostra incluiu 513 pacientes do grupo de testes seletivos e 512 pacientes do grupo de testes rotineiros. Os dois grupos tiveram proporções similares de pacientes que faltaram à cirurgia e não a remarcaram ( $2 \%$ em cada grupo $=10$ pacientes) .

\begin{tabular}{|c|c|}
\hline \multicolumn{2}{|c|}{$\begin{array}{l}\text { Indicações para realização de hemoglobina sangüínea, glicemia de jejum } \\
\text { e eletrocardiograma. Brasil, } 2001 .\end{array}$} \\
\hline Exame & Indicações \\
\hline \multirow[t]{9}{*}{ Hemoglobina sangüínea } & Anemia sintomática \\
\hline & Distúrbio da hemostasia \\
\hline & Estados de hipercoagubilidade \\
\hline & Uso de anticoagulante ou corticosteróide \\
\hline & Câncer \\
\hline & Nefropatia \\
\hline & Quimioterapia \\
\hline & Radioterapia \\
\hline & Síndromes de má absorção \\
\hline \multirow[t]{12}{*}{ Glicemia de jejum } & Diabetes \\
\hline & Cetoacidose diabética \\
\hline & Coma hiperosmolar \\
\hline & Acidose láctica \\
\hline & Hipoglicemia sintomática \\
\hline & Síndrome de Cushing \\
\hline & Doença de Addison \\
\hline & Obesidade mórbida \\
\hline & Desnutrição \\
\hline & Síndrome de má absorção \\
\hline & Acidente vascular cerebral \\
\hline & Uso de corticosteróide \\
\hline \multirow[t]{13}{*}{ Eletrocardiograma } & Cardiopatias congênitas \\
\hline & Doenças valvulares cardíacas \\
\hline & Doença cardíaca coronariana \\
\hline & Arritmias \\
\hline & Distúrbios de condução \\
\hline & Insuficiência cardíaca \\
\hline & Miocardites e miocardiopatias \\
\hline & Febre reumática \\
\hline & Doenças do pericárdio \\
\hline & Hipertensão pulmonar \\
\hline & Hipertensão arterial \\
\hline & Doença vascular cerebral ou periférica \\
\hline & Diabetes \\
\hline
\end{tabular}

Os tipos de complicações clínicas intra-operatórias e a freqüência com que ocorreram foram semelhantes nos dois grupos $(9,6 \%$ no grupo de testes rotineiros e $9,7 \%$ no grupo de testes seletivos - p 0,923 - Tabela 4).

Foram realizados 604 exames no grupo de testes seletivos e 1.536 exames no grupo de testes rotineiros, com média de exames por pacientes de respectivamente 1,18 e 3,00. O gasto com a realização dos exames de hemoglobina, glicemia de jejum e eletrocardiograma dos 512 pacientes do grupo de testes rotineiros foi de 
Tabela 2

Características dos pacientes com indicação de facectomia, de acordo com o grupo. Campinas, São Paulo, Brasil, 2001.

\begin{tabular}{lccc}
\hline & $\begin{array}{c}\text { Testes rotineiros } \\
(\mathrm{n}=512)\end{array}$ & $\begin{array}{c}\text { Testes seletivos } \\
(\mathrm{n}=513)\end{array}$ & $\mathrm{p}$ \\
\hline Idade (anos) & $66,4 \pm 11,9$ & $66,7 \pm 11,3$ & $0,714 \mathrm{a}$ \\
\hline & \multicolumn{3}{c}{ Número de pacientes (\%) } \\
\hline $\begin{array}{l}\text { Sexo masculino } \\
\text { American Society }\end{array}$ & $279(54,5)$ & $268(52,2)$ & $0,510 \mathrm{~b}$ \\
1 & $71(13,9)$ & $76(14,8)$ & $0,850 \mathrm{~b}$ \\
2 & $394(76,9)$ & $387(75,5)$ & \\
3 & $47(9,2)$ & $50(9,7)$ & \\
\hline
\end{tabular}

a one-way ANOVA

b qui quadrado (YATES)

Tabela 3

Características dos grupos de acordo com a indicação de exames pré-operatórios em facectomias. Campinas, São Paulo, Brasil, 2001.

\begin{tabular}{lccc}
\hline Tipo de exame & $\begin{array}{c}\text { Testes rotineiros } \\
(n=512)\end{array}$ & $\begin{array}{c}\text { Testes seletivos } \\
(n=513)\end{array}$ & p \\
\hline $\begin{array}{l}\text { Hemoglobina } \\
\text { Com indicação }\end{array}$ & 18 & 19 & 1,000 a \\
Sem indicação & 494 & 494 & 0,582 a \\
Glicemia & 113 & & \\
Com indicação & 399 & 105 & \\
Sem indicação & & 408 & 0,617 a \\
Eletrocardiograma & 474 & 480 & \\
Com indicação & 38 & 33 & \\
Sem indicação & & & \\
\hline
\end{tabular}

a qui quadrado (YATES) ria dos pacientes submetidos a facectomia 9,11, 41, constituía uma população de baixo risco cirúrgico 42,43 . Hollenberg 44 classifica esse procedimento entre os de menor risco de eventos cardíacos (risco de morte ou infarto agudo do miocárdio menor que 1\%), assim como o são endoscopia, cirurgia de mama e operações dermatológicas. Backer et al. 45 observaram que a incidência de reinfarto do miocárdio em facectomias com anestesia local era próxima a zero, não havendo risco adicional à cirurgia mesmo nesse grupo de pacientes cardíacos.

Na população participante deste estudo, de acordo com os critérios pré-estabelecidos (Tabela 1), a dosagem de hemoglobina seria desnecessária em $96,4 \%$ dos pacientes. Sabe-se que anemia moderada (hemoglobina $>10 \mathrm{mg} / \mathrm{dl}$ ) não aumenta os riscos relacionados à cirurgia, e geralmente deve-se à deficiências de ferro, ácido folínico e/ou vitamina B12 46,47. Já anemias severas $(\mathrm{Hb}<8 \mathrm{mg} / \mathrm{dl})$ estão associadas a maior mortalidade operatória e morbidade cardíaca, mas as mesmas são, na sua maioria, clinicamente detectáveis 48 Portanto, nas cirurgias com pouco sangramento, como facectomias, não é necessária a dosagem de hemoglobina, a não ser que o paciente tenha uma his tória de anemia com repercussões clínicas 21 .

O exame de glicemia seria dispensável em $78,7 \%$ dos pacientes, segundo os critérios listados no Tabela 1, entretanto, mesmo nos casos em que há uma justificativa baseada na literatura, a solicitação desse exame é questionável 21,49 . Tal dúvida se deve ao fato de que a quase totalidade das solicitações deriva da presença de diabetes, e existem mitos referentes à associação entre diabetes e cirurgia, pois, na tentativa de evitar complicações cirúrgicas relacionadas à diabetes, como maior propensão à infecção e à deiscência de suturas, valoriza-se de forma demasiada o controle da glicemia na época da cirurgia, quando, na verdade, o mais importante é o controle crônico 47,50,51. Aliás, uma hiperglicemia moderada (até $250 \mathrm{mg} / \mathrm{dl}$ ) no perioperatório é preferível à possibilidade de ocorrer hipoglicemia 52 . Some-se a isso o fato de que o estresse diante da perspectiva de cirurgia pode impedir que pacientes previamente controlados atinjam valores agudos considerados ideais e já alcançados historicamente no seu controle rotineiro 47 .

O eletrocardiograma teve indicação clínica em $93,1 \%$ dos pacientes. Anormalidades no ECG são comuns, variando de $14 \%$ a $64 \%$, e a freqüência de anormalidades aumenta com a idade 53,54. Como anormalidades no ECG são freqüentes, o médico, se não solicitar um ECG pré-operatório, não poderá determinar se as 
anormalidades no ECG pós-operatórias são novas 21,55 . Todavia, não há consenso quanto à necessidade de ECG pré-operatório de rotina em pacientes adultos. O ECG rotineiro tem como conseqüência um grande número de resultados falso-positivos, que podem levam a procedimentos adicionais com ônus para a eficiência do serviço prestado à população. O maior efeito positivo do ECG pré-operatório de rotina é detectar IAM não diagnosticado clinicamente, cujo risco aumenta com a idade; contudo, mesmo no grupo de mais alto risco de IAM (homens acima de 75 anos), a incidência estimada de infarto nos seis meses precedentes a cirurgia é relativamente baixa $(<0.5 \%) 23,56,57$.

Rabkin \& Horne 58 avaliaram 812 pacientes que tinham estudo eletrocardiográfico prévio para determinar se novas anormalidades no ECG pré-operatório de rotina resultavam em alteração na conduta dos pacientes cirúrgicos. Os resultados mostraram mudança na conduta em apenas dois pacientes. Gold et al. 59 obtiveram resultados semelhantes em 751 pacientes submetidos à cirurgia ambulatorial.

Tomando-se por base o número total de facectomias pagas pelo SUS (264.406), no Brasil, no ano de 2001, foram gastos $\mathrm{R} \$ 2.908 .466,00$ em exames pré-operatórios 38 . Como o grupo de testes seletivos realizou $60,7 \%$ menos exames que o grupo de testes rotineiros, com a solicitação seletiva desses exames poderíamos ter economizado R\$ 1.765.438,86, sem repercussões do ponto de vista de complicações clínicas intra-operatórias para o paciente. Esse valor equivale ao pagamento de outras 3.985 cirurgias de catarata, mais do que o número total de cirurgias realizadas no Estado do Amazonas, ou no Espírito Santo, pelo SUS no mesmo período 38. Esses recursos poderiam ser utilizados nos locais onde há fila de espera ou em outras ações de saúde. Outro aspecto mais abrangente que a economia dos recursos em si, é que a solicitação seletiva de exames é uma forma de diminuir as barreiras para a cirurgia, uma vez que exames desnecessários podem significar também mais idas ao hospital e adiamento desnecessário de cirurgias 16.

Deve-se salientar que a rotina de exames pré-operatórios nesse serviço já é menor do que na maioria dos outros por haver uma constante preocupação com a economia e otimização dos recursos disponíveis 60,61. Para serviços que têm rotinas de exames mais amplas, incluindo exames de urina, coagulação sanguínea e eletrólitos essa racionalização será ainda maior.

A análise econômica em saúde tem importância cada vez maior, uma vez que os recursos
Tabela 4

Tipos de complicações clínicas intra-operatórias ocorridas em facectomias, de acordo com o grupo. Campinas, São Paulo, Brasil, 2001.

\begin{tabular}{lrr}
\hline Evento & \multicolumn{2}{c}{ Número de pacientes $(\%)$} \\
Testes rotineiros & $\begin{array}{c}\text { Testes seletivos } \\
(n=502)\end{array}$ & $43(8,5)$ \\
\hline Hipertensão & $41(8,2)$ & - \\
Arritmia & $1(0,2)$ & $1(0,2)$ \\
Angina & - & - \\
Ataque isquêmico transitório & $1(0,2)$ & $3(0,6)$ \\
Broncoespasmo & $3(0,6)$ & $2(0,4)$ \\
Ansiedade & $2(0,4)$ & $49(9,7)$ \\
Total & $48(9,6)$ & \\
\hline
\end{tabular}

estão mais escassos e há uma competição por setores de diferentes necessidades. Para que os administradores da saúde pública possam fazer escolhas adequadas, é necessário ter-se conhecimento dos custos e suas possíveis alternativas. Sem análise sistemática é difícil identificar claramente as alternativas relevantes 62 .

Este estudo tem algumas limitações. Foram registradas apenas morbidades clinicamente significantes; por exemplo, extra-sístoles atriais isoladas e outras arritmias menores não foram incluídas. Outra limitação foi a adoção de uma rotina relativamente sucinta para os pacientes do grupo de testes rotineiros, resumindo-se a três testes: eletrocardiograma com 12 derivações, dosagem de hemograma e dosagem da glicemia de jejum. Porém, esses exames são os mais requisitados em facectomias e, além do mais, compunham a rotina padrão do centro médico acadêmico onde o estudo foi realizado 60,61 . Outra limitação foi que os eventos médicos relacionados à cirurgia limitaram-se ao período pós-operatório que se estendia até a alta do paciente no mesmo dia da intervenção. Todavia, este período inclui mais de $75 \%$ dos eventos cardiopulmonares que ocorrem na primeira semana de pós-operatório 9. É importante enfatizar que o foco de nosso estudo foi uma população relativamente saudável submetida a um procedimento de baixo risco 63 .

Os resultados sugerem que, dentro da rotina de um hospital universitário de referência, no Brasil, a requisição seletiva de exames préoperatórios sistêmicos em facectomias, além de não prejudicar o paciente do ponto de vista de complicações clínicas intra-operatórias, possibilita expressiva economia de recursos quando comparada à atual rotina de requisição de exames. 


\section{Resumo}

O objetivo deste experimento clínico foi determinar a freqüência dos exames considerados dispensáveis numa rotina pré-operatória de facectomias e avaliar os gastos com eles. Se o paciente foi sorteado para testesseletivos, solicitou-se que nenhum teste fosse executado, a menos que o paciente apresentasse um novo problema médico, ou piora de uma doença preexistente, a qual requeresse os testes independentemente da realização da cirurgia. Para pacientes sorteados para testes-rotineiros,foram solicitados: eletrocardiograma, dosagem de hemoglobina e glicemia de jejum. Foram calculados os gastos com exames considerados desnecessários. A amostra de 1.025 pacientes incluiu 513 sorteados para testes seletivos e 512 para testes rotineiros. A freqüência de eventos adversos intra-operatórios foi semelhante nos dois grupos ( $p=0,923)$. O grupo de testes seletivos realizou de $60,7 \%$ menos exames que o de testes rotineiros. Os resultados sugerem que a requisição seletiva de exames pré-operatórios em facectomias, além de não prejudicar o paciente do ponto de vista de complicações clínicas intra-operatórias,possibilita expressiva economia de recursos quando comparada à atual rotina.

Catarata; Idoso; Exames Médicos; Cuidados Pré-operatórios

\section{Colaboradores}

C. E. L. Arieta contribui na concepção e desenho de estudo, na análise, interpretação de dados e revisão e aprovação para versão final. M. A. Nascimento e R. P. C. Lira colaboraram na concepção do estudo, levantamento de dados, análise e interpretação de dados, e revisão final. N. Kara-José participou da concepção do estudo, interpretação de dados e revisão final do artigo.

\section{Agradecimentos}

Ao apoio da Fundação de Amparo à Pesquisa do Estado de São Paulo, por intermédio de Bolsa de Mestrado processo oㅡ 00/02373-9.

\section{Referências}

1. Apple DJ, Ram J, Foster A, Peng Q. Elimination of cataract blindness: a global perspective entering the new millenium. Surv Ophthalmol 2000; 45 Suppl 1:S1-196.

2. Foster A, Johnson G. Blindness in the developing world. Br J Ophthalmol 1993; 77:398-9.

3. Dawson CR, Schwab IR. Epidemiology of cataract - a major cause of preventable blindness. Bull World Health Organ 1981; 59:493-501.

4. Schwab L. Eye care in developing nations. $3^{\text {th }}$ Ed. San Francisco: The Foundation of the American Academy of Ophthalmology; 1999.

5. Blay SL. Envelhecimento populacional: panorama demográfico. J Bras Psiquiatr 1991; 40:361-4.

6. Wilson J. Clearing the cataract backlog. $\mathrm{Br} \mathrm{J}$ Ophthalmol 1987; 71:158-60.

7. Duke-Elder S. System of ophthalmology. London: Henry Kimpton; 1969.

8. Schein OD. Assessing what we do: the example of preoperative medical testing. Arch Ophthalmol 1996; 114:1129-31.

9. Schein OD, Katz J, Bass EB, Tielsch JM, Lubonski LH, Feldman MA, et al. The value of routine preoperative medical testing before cataract surgery. N Engl J Med 2000; 342:168-75. 
10. Bellan L. Preoperative testing for cataract surgery. Can J Ophthalmol 1994; 29:111-4.

11. Breslin PP. Mortality in ophthalmic surgery. Int Ophthalmol Clin 1973; 13:215-26.

12. Linebarger EJ, Hardten DR, Shah GK, Lindstrom RL. Phacoemulsication and modern cataract surgery. Survey Ophthalmol 1999; 44:123-47.

13. Rosenfeld SI, Litinky SM, Snyder DA, Plosker H, Astrove AW, Schiffman, J. Effectiveness of monitored anesthesia care in cataract surgery. Ophthalmology 1999; 106:1256-60.

14. Foster A. Cataract - a global perspective: output, outcome and outlay. Eye 1999; 13:449-53.

15. Kara-José N, Arieta CEL, Temporini ER, Kang KM, Ambrósio LE. Tratamento cirúrgico de catarata senil: óbices para o paciente. Arq Bras Oftalmol 1996; 59:573-7.

16. Kara-José N, Almeida GV, Alves MR, Kikuta HS, Arieta CEL. Campanha nacional de prevenção de cegueira e reabilitação visual do idoso - 1996. Rev Assoc Med Bras 1997; 76:293-6.

17. Ungaro ABS, Vilella FF, Klein RCA, Kara-José N, Alves MR, Cresta FB. Campanha nacional de prevenção da cegueira e campanha nacional de reabilitação visual do idoso: experiência no Hospital das Clinicas da Faculdade de Medicina da Universidade de São Paulo. Rev Assoc Med Bras 1997; 76:97-100.

18. Steinberg EP. Content and costs of cataract surgery. Arch Ophthalmol 1993; 111:1041-9.

19. Lira RPC, Covolo GA, Monsanto AR, Kara-José N, Arieta CEL. Influence of preoperative testing on cancellation of ambulatory cataract surgery in adults. Ann Ophthalmol 2002; 34:203-5.

20. Wordmald R. Cataract surgery - quantity and quality. Br J Ophthalmol 1999; 83:889-91.

21. MacPherson DS. Preoperative laboratory testing: should any tests be "routine" before surgery? Med Clin North Am 1993; 77:289-308.

22. Blery C, Szatan M, Fourgeaux B, CharpakY, Darne B, Chastang CL, et al. Evaluation of a protocol for selective ordering of preoperative tests. Lancet 1986; 1:139-41.

23. Goldberger AL, O'Konski M. Utility of the routine electrocardiogram before surgery and on general hospital admission. Ann Intern Med 1986; 105: 552-7.

24. Johnson H, Knee-Loli S, Butler TA, Munoz E, Wise L. Are routine preoperative laboratory screening tests necessary to evaluate of ambulatory surgical patients? Surgery 1988; 104:639-45.

25. Kaplan EB, Boeckmann AJ, Roizen MF, Sheiner LB. Elimination of unnecessary preoperative laboratory tests. Anesthesiology 1982; 57 Suppl 3: 445.

26. Kaplan EB, Sheiner LB, Boeckmann AJ, Roizen MF, Beal ST, Cohen SN, et al. The usefulness of preoperative laboratory screening. JAMA 1985; 253:3576-81.

27. Brandspigel K, City E. Cost-effective preoperative laboratory testing. JAMA 1994; 271:319-20.

28. Nardella A, Pechet L, Snyder M. Continuous improvement, quality control, and cost containment in clinical laboratory testing. Arch Pathol Lab Med 1995; 119:518-22.

29. Macario A, Roizen MF, Thisted RA, Kim S, Orkin
FK, Phelps C. Reassessment of preoperative laboratory testing has changed the test-ordering patterns of physicians. Surg Gynecol Obstet 1992; 175:539-47.

30. Mancuso CA. Impact of new guidelines on physicians' ordering of preoperative tests. J Gen Intern Med 1999; 14:166-72.

31. Monteiro JV. Campanha nacional de cirurgias de catarata 2000. Jornal Oftalmológico Jota Zero 2000; 74:4-5.

32. Kara-José N, Arieta CEL. South American programme: Brazil. Community Eye Health 2000; 15: 55-7.

33. Jaffe NS. Atlas of ophthalmic surgery. 2nd ed. Barcelona: Mosby-Wolfe; 1996.

34. American Society of Anesthesiologists. New classification of physical status. Anesthesiology 1963; 24:111.

35. Lira RPC, Nascimento MA, Moreira Filho DC, Kara-José N, Arieta CEL. Are routine preoperative medical tests needed with cataract surgery? Rev Panam Salud Publica 2001; 10:13-7.

36. Tierney Jr LM, McPhee SJ, Papadakis MA. Current medical diagnosis and treatment. 38th ed. Stanford: Appleton \& Lange, 1999.

37. Fischer SP. Cost-effective preoperative evaluation and testing. Chest 1999; 115 Suppl 5:96S-100S.

38. Departamento de Informática do SUS. Número de facectomias realizadas pelo SUS em 2001. <http: //www.datasus.gov.br (acessado em 2/Ago/ 2002).

39. Daniel WW. Biostatistics: a foundation for analysis I the health sciences. 7 th ed. New York: Wiley; 1999.

40. Fleiss JL. Statistical methods for rates and proportions. 2nd ed. New York: Wiley; 1981.

41. Ferreira AA, Katayama M. Anestesia em oftalmologia. Rev Bras Anestesiol 1981; 31:481-95.

42. Menke H, Klein A, John KD, Junginger T. Predictive value of ASA classification for the assessment of the perioperative risk. Int Surg 1993; 78:266-70.

43. Prause G, Ratzenhofer-Comenda B, Pierer G, Smole-Jüttner F, Glanzer H, Smolle J. Can ASA grade or Goldman's cardiac risk index predict perioperative mortality? Anesthesia 1997; 52:203-6.

44. Hollenberg SM. Preoperative cardiac risk assessment. Chest 1999; 115 Suppl 5:51S-57S.

45. Backer CL, Tinker JH, Robertson DM, Vliestra RE. Myocardial reinfarction following local anesthesia for ophthalmic surgery. Anesth Analg 1980; 59:257-62.

46. Linker CA. Blood. In: Tierney Jr LM, McPhee SJ, Papadakis MA, editors. Current medical diagnosis and treatment. 38th ed. Stanford: Appleton \& Lange; 1999. p. 485-537.

47. Siperstein MD. Special medical problems in surgical patients. In: Way LW, editor. Current surgical diagnosis and treatment. 10th ed. Stanford: Appleton \& Lange; 1994. p. 40-2.

48. Carson JL, Poses RM, Spence RK, Bonavita G. Severity of anemia and operative mortality and morbidity. Lancet 1988; 1:727-9.

49. Meyer P, Roizen MF, Murray W, Gronert G, Grogono A, Duke P. Who needs an ECG or a blood glucose test preoperatively: use of technology to improve selection. Anesthesiology, 75 Suppl 3A: A441. 
50. Eckersley JRT, Dudley HAF. Wounds and wound healing. Br Med Bull 1988; 44:423-36.

51. Hjortrup A, Rasmussen BF, Kehlet H. Morbidity in diabetic and non-diabetic patients after major vascular surgery. Br Med J 1983; 287:1107-9.

52. Hjortrup A, Dyremose E, Hjorts NC, Kehlet H. Influence of diabetes mellitus on operative risk. Br J Surg 1985; 72:783-5.

53. Jakobsson A, White T. Routine preoperative electrocardiograms. Lancet 1984; 1:972.

54. Nascimento Jr P, Castiglia YMM. O eletrocardiograma como exame pré-operatório do paciente sem doença cardiovascular. É mesmo necessário? Rev Bras Anestesiol 1998; 48:352-61.

55 . Velanovich $\mathrm{V}$. The value of routine preoperative laboratory testing in predicting postoperative complications: a multivariate analysis. Surgery 1991; 109:236-43.

56. Kannel WB, Abbott RD. Incidence and prognosis of unrecognized myocardial infarction: An update on the Framingham study. N Engl J Med 1984; 311:1144-7.

57. Rao TLK, Jacobs KH, El-Etr AA. Reinfarction following anesthesia in patients with myocardial infarction. Anesthesiology 1983; 59:499-505.
58. Rabkin SW, Horne JM. Preoperative electrocardiography: effect of new abnormalities on clinical decisions. Can Med Assoc J 1983; 128:146-7.

59. Gold BS, Young ML, Kinman JL, Kitz DS, Berlin J, Schwatz JS. The utility of preoperative electrocardiograms in the ambulatory surgical patient. Arch Intern Med 1992; 152;301-5, 1992.

60. Arieta CEL, Kara-José N, Carvalho Filho DM, Alves MR. Optimization of an university cataractpatient care service in Campinas, Brazil. Ophthalmic Epidemiol 1999; 6:1-10.

61. Bass EB, Steinberg EP, Luthra R, Schein OD, Tielsch JM, Javitt JC, et al. Do ophthalmologists, anesthesiologists, and internists agree about preoperative testing in healthy patients undergoing cataract surgery? Arch Ophthalmol 1995; 113: 1248-56.

62. Drummond MF. Methods for the economic evaluation of Health care programmes. 2nd Ed. London: Oxford Medical Publications; 1997.

63. Carter DC, Campbell D. Evaluation of the risks of surgery. Br Med Bull 1988; 44:322-40.

Recebido em 11/Out/2002

Versão final reapresentada em 30/Mai/2003 Aprovado em 16/Out/2003 